\title{
Neonatal Seizures do not Exclude Dravet Syndrome Diagnosis
}

\author{
Elżbieta Szczepanik ${ }^{1}$, Iwona Terczyńska ${ }^{1 *}$, Dorota Antczak-Marach ${ }^{1}$, Paulina Górka-Skoczylas ${ }^{1,2}$ and Dorota Hoffman-Zacharska ${ }^{2}$ \\ ${ }^{1}$ Clinic of Neurology of Children and Adolescents, Institute of Mother and Child, Warsaw, Poland \\ ${ }^{2}$ Dept. of Medical Genetics; Institute of Mother and Child, Warsaw, Poland
}

*Corresponding author: Iwona Terczyńska, Clinic of Neurology of Children and Adolescents, Institute of Mother and Child, Kasprzaka 17A, 01-211 Warsaw, Poland, Tel: +48 223277 307; Fax: +48 223277 130; E-mail: iwonaterczynska@onet.eu

Received date: January 18, 2016; Accepted date: March 09, 2016; Published date: March 16, 2016

Copyright: (c) 2016 Szczepanik E, et al. This is an open-access article distributed under the terms of the Creative Commons Attribution License, which permits unrestricted use, distribution, and reproduction in any medium, provided the original author and source are credited.

\begin{abstract}
Introduction: We present a patient with Dravet syndrome (DS) caused by the SCN1A mutation, and with neonatal seizure history.
\end{abstract}

Material and Methods: Patient medical history was reviewed with focus on onset age, seizure types, EEG features, neuroimaging studies and treatment response.

Results: A 16-month-old boy with an abnormal perinatal period presented a seizure on the second day of life during febrile congenital infection. He had developed focal right-sided seizures with sensitivity to low-grade fever since 4 months of age. The SCN1A nonsense mutation was confirmed. Subsequent disease course with multiple seizure type's development became consistent with DS clinical diagnosis.

Conclusions: DS diagnosis should not be ruled out in patients with neonatal seizures who meet the clinical, EEG and evolution criteria for this syndrome.

Keywords: Neonatal seizures; Febrile seizures; Dravet syndrome; SCN1A

\begin{abstract}
Abbreviations
CLB-Clobazam; CPS-Complex Partial Seizures; CSF-Cerebrospinal Fluid; DS-Dravet Syndrome; FS-Febrile Seizures; GTCS-Generalized Tonic-clonic Seizures; IPS-Intermittent Photic Stimulation; LEVLevetiracetam; PB-Phenobarbital; VPA-Valproate

\section{Introduction}

Dravet syndrome (DS) core phenotype consists of refractory multiple seizure types such as focal, hemiclonic with alternating side, tonic-clonic, myoclonic seizures, and absences, often complicated by status epilepticus [1]. The most typical feature of this syndrome is that seizures can be provoked by hyperthermia [2].
\end{abstract}

Seizures usually start during the first year of life in normally developing children. DS is associated with cognitive and behavioral disorders in addition to epilepsy. Based on our experience of enhanced syndrome clinical spectrum allows us to suspect DS in less and less typical clinical conditions, even exceeding well known borderline cases [2].

Here, we present a 16-month-old boy with DS caused by a $S C N 1 A$ mutation, and with neonatal seizure history. The appearance of these seizures made the correct diagnosis more challenging and complicated. According to our best knowledge no case of DS with neonatal seizures has been described so far.

\section{Case report}

The boy was the 1st child of non-consanguineous young healthy parents. The family history was negative towards epilepsy and febrile seizures (FS), and other CNS diseases. Pregnancy was complicated by diarrhoea in the $36^{\text {th }}$ week of pregnancy. He was born at 42 -week gestation, with weight $3400 \mathrm{~g}$, and head circumference $34 \mathrm{~cm}, 9 / 9$ points in Apgar scale. However, intrauterine infection and serological conflict were diagnosed. Neonatal clonic seizure, followed by bradycardia, occurred on the 2nd day of life during slightly elevated body temperature $\left(37.5^{\circ} \mathrm{C} / 99.5^{\circ} \mathrm{F}\right)$. Head USG and CSF were normal. TORCH infection was negative. EEG was not performed. Repeated neurological and cardiologic assessments were within the normal range.

Succeeding seizures had been observed since 4 months of life, always during infections with low-grade fever $\left(37.5-38^{\circ} \mathrm{C} / 99.5-\right.$ $100.4^{\circ} \mathrm{F}$ ) or after sun bathing. Simple motor right-sided seizures with Todd's paresis, complex partial seizures (CPS) with right head turn, and generalized tonic-clonic seizures (GTCS) with right side predominance were observed. The first EEG at 5 months of age showed no interictal epileptiform activity but lack of spindles. Brain CT scan was normal. PB up to $60 \mathrm{mg} /$ day and later with VPA in dose 200 $\mathrm{mg} /$ day were introduced without improvement and the child experienced GTCS and CPS status epilepticus, both lasting 40 minutes despite rectal diazepam administration.

The boy was admitted to our clinic at 7.5 months of age. Psychomotor development was normal apart from slightly delayed gross motor skills. Metabolic screening and brain MRI scans were normal. Interictal EEG showed focal spikes in posterior areas. IPS was not performed. Considering the etiology, we were taking into account abnormal perinatal history with neonatal seizure, and focal seizures 
with right-sided dominance which could be in favor of symptomatic epilepsy. On the other hand, such numerous seizures with low-grade fever sensitivity, and tendency to status epilepticus might have suggested SCN1A channelopathy. While waiting for the result of genetic testing, we decided to avoid sodium channel blocker antiepileptic drugs. PB was replaced with LEV, showing some clinical benefit. The subsequent disease course revealed also myoclonic and absence seizures probably. Ictal EEG at 10 months of age showed alternating hemiclonic seizures with paroxysms of spikes and spikewave complexes. At that time, based on the clinical background and ictal EEG, we considered the diagnosis of DS.

The SCN1A gene molecular analysis was completed when the boy was 11 months old and revealed a heterozygous truncating mutation presence in exon 19 c.[3734_3735insTGATCAGC];[=] (p. [Arg1245Leufs $\left.\left.{ }^{*} 28\right] ;[=]\right)$. Such $S C N 1 A$ nonsense mutations causing a premature termination of protein synthesis in the region of DIIIS1-S2 linker and haploinsufficiency of the Nav1.1 protein are the cause of epileptic encephalopathy-Dravet Syndrome (SCN1A Variant Database; http://www.molgen.ua.ac.be/SCN1AMutations). SCN1A gene exon 19 analysis performed for the proband's parents showed that his father was a mutation carrier. DNA derived from leukocyte analysis showed somatic mosaicism at a low level $<10 \%$ c. $[=/$ 3747_3735insTGATCAGC];[=]. The family was referred to the Genetic Counselling Unit, because of DS recurrence risk in the subsequent pregnancies.

At the end of our observation, the 16-month-old boy was treated with VPA, CLB and stiripentol, having rare short CPSs and GTCSs. Psychomotor development remained within the normal range, although motor gross skills seemed to be delayed (he did not walk independently). Neurological examination, except for mild hypotonia, was normal. His interictal sleep EEG was normal at that time.

\section{Discussion}

We present a Dravet syndrome case with a confirmed SCN1A mutation inherited from an asymptomatic father. Abnormal perinatal history with neonatal seizure and focal seizures being the only seizure type at that time, first suggested symptomatic/ structural epilepsy etiology. However, the strong sensitivity to hyperthermia directed us to consider SCN1A channelopathy as the background condition. Exclusive focal seizures appearance had not excluded such possibilities, as subjects with focal epilepsies, according to Lossin et al. might constitute $1.1 \%$ of epilepsy caused by $S C N 1 A$ mutations [3]. The subsequent disease course with multiple seizure types development became consistent with DS clinical diagnosis.

Seizures, predominantly febrile ones, start in DS during the first 1214 months of life, mostly in the first 6 months. The earliest seizure onset described in a DS patient was the second month of life [4]. Neonatal seizure in the presented case occurred during congenital infection with slightly elevated body temperature. Nevertheless, we cannot call it a febrile seizure, because this type of seizure is defined as "a seizure occurring in childhood after one month of age..." according to the International League Against Epilepsy [5]. This definition states that febrile seizures are without previous neonatal seizures or a previous unprovoked seizure, and not meeting criteria for other acute symptomatic seizures". To be in line with the above definition, we should have not called seizures occurring during hyperthermia in our infant patient as febrile seizures but rather epileptic seizures provoked by hyperthermia. This example shows how difficult are sometimes to comply with established guidelines.

Trying to classify the neonatal seizures in our patient, we could only speculate whether it was an acute (symptomatic) one, occurring due to an abnormal perinatal period just in a child with Dravet syndrome or whether it was the first seizure of DS during the neonatal period due to combined effect of environmental and genetic factors. In favor of the latter hypothesis could be growing evidence that other genetic and / or environmental factors may play a role in epileptic phenotype modification in patients with the $S C N 1 A$ mutation [6,7]. In agreement with the first possibility, there are papers showing coexistence of two etiologically different seizure disorders / epileptic syndromes in the same subject $[8,9]$.

Mutations in the SCN1A gene cause a wide spectrum of seizure disorders, ranging from early-onset isolated febrile seizures to Dravet syndrome which is the most severe phenotype (OMIM \#604403). So far, several hundred patients with DS and even many more with GEFS + due to the SCN1A mutation have been reported. We have not found a case among them with neonatal seizures nor epilepsy with neonatal onset. This raises a question if individuals with SCN1A mutation are not prone to seizures in the neonatal period. One possible explanation might be connected with the human neuronal inhibitory system developmental aspects. As Kanaumi et al. (2008) report, $K C N Q$ channels constitute a predominantly inhibitory system in the youngest neonates, whereas GABA-ergic transmission takes over the main inhibitory function later on [10]. However, GABAA receptors activation causes membrane depolarization in the first postnatal weeks, rather than hyperpolarization typical for mature GABA-ergic synapses. This process is related to maturation changes in the transmembrane chloride ion gradient [11]. Thus we can discuss if GABA-ergic interneurons dysfunction due to $S C N 1 A$ mutation resulting in increased susceptibility to seizures in post neonatal age, might diminish it during the neonate period. In the presented case, those age-dependent neurotransmission properties could have been modulated by perinatal events resulting in neonatal seizure. If so, we cannot consider neonatal seizures as a part of DS phenotype. However, this does not mean such cases, as ours, do not occur in everyday practice. They can go underdiagnosed and might be considered as symptomatic epilepsy, as we did at the beginning of our observation. There is no need to stress that misdiagnosis of DS may be followed by improper treatment resulting in seizure exacerbation and cognitive worsening.

\section{Conclusions}

The aim of the presented paper is to share our experience with an unusual case showing that neonatal seizure occurrence does not exclude diagnosis of DS. Breaking our stereotypes in diagnosis of this well-defined epileptic syndrome had let us to reconsider initial diagnosis and to show that we should take into account the possible additional effect of different phenotype modifiers in any case with atypical clinical picture. Appropriate syndromic classification let us to perform molecular diagnosis and to correct management of the disease, and genetic counselling.

\section{Acknowledgments}

This work was supported by grant from MSHE/NSC NN407054439. 
Citation: Szczepanik E, Terczynska I, Antczak-Marach D, Górka-Skoczylas P, Hoffman-Zacharska D (2016) Neonatal Seizures do not Exclude Dravet Syndrome Diagnosis. J Neurol Neurophysiol 7: 360. doi:10.4172/2155-9562.1000360

Page 3 of 3

\section{References}

1. Engel J (2001) A proposed diagnostic scheme for people with epileptic seizures and with epilepsy: report of the ILAE Task Force on Classification and Terminology. Epilepsia 42: 796-803.

2. Caraballo RH, Fejerman N (2006) Dravet syndrome: a study of 53 patients. Epilepsy Res 70: S231-238.

3. Lossin C (2009) A catalog of SCN1A variants. Brain Dev 31: 114-130.

4. Xu X, Zhang Y, Sun H, Liu X, Yang X, et al. (2014) Early clinical features and diagnosis of Dravet syndrome in 138 Chinese patients with SCN1A mutations. Brain Dev 36: 676-681.

5. (1993) Guidelines for epidemiologic studies on epilepsy. Commission on Epidemiology and Prognosis, International League Against Epilepsy. Epilepsia 34: 592-596.

6. Singh NA, Pappas C, Dahle EJ, Claes LR, Pruess TH, et al. (2009) A role of $S C N 9 A$ in human epilepsies, as a cause of febrile seizures and as a potential modifier of Dravet syndrome. PLoS Genet 5: e1000649.
7. Craig AK, de Menezes MS, Saneto RP (2012) Dravet syndrome: patients with co-morbid SCN1A gene mutations and mitochondrial electron transport chain defects. Seizure 21: 17-20.

8. Pisani F, Facini C, Pavlidis E, Spagnoli C, Boylan G (2015) Epilepsy after neonatal seizures: literature review. Eur J Paediatr Neurol 19: 6-14.

9. Koutroumanidis M, Hennessy MJ, Elwes RD, Binnie CD, Polkey CE (1999) Coexistence of temporal lobe and idiopathic generalized epilepsies. Neurology 53: 490-495.

10. Kanaumi T, Takashima S, Iwasaki H, Itoh M, Mitsudome A, et al. (2008) Developmental changes in KCNQ2 and KCNQ3 expression in human brain: possible contribution to the age-dependent etiology of benign familial neonatal convulsions. Brain Dev 30: 362-369.

11. Sanchez RM, Jensen FE (2001) Maturational aspects of epilepsy mechanisms and consequences for the immature brain. Epilepsia 42: 577-585. 